International Journal of Pure and Applied Mathematics

Volume 100 No. 3 2015, 347-360

ISSN: 1311-8080 (printed version); ISSN: 1314-3395 (on-line version)

url: http://www.ijpam.eu

doi: http://dx.doi.org/10.12732/ijpam.v100i3.1

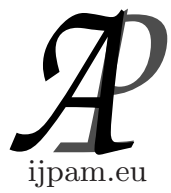

\title{
TOPOLOGICAL PROPERTIES OF $L$-FUZZY UNIFORM SPACES
}

\author{
A.A. Ramadan ${ }^{1}$, E.H. Elkordy ${ }^{2}$, Yong Chan $\mathrm{Kim}^{3} \S$ \\ ${ }^{1,2}$ Department of Mathematics \\ Faculty of Science \\ Beni-Suef University, Beni-Suef, EGYPT \\ ${ }^{3}$ Department of Mathematics \\ Gangneung-Wonju University \\ Gangneung, Gangwondo 210-702, KOREA
}

\begin{abstract}
In this paper, we define $L$-neighborhood spaces and investigated the topological properties of $L$-fuzzy uniformity in complete residuated lattices. We obtain $L$-fuzzy topology and $L$-neighborhood spaces induced by $L$ fuzzy uniformity. Moreover, we study the relations among $L$-fuzzy topology, $L$-neighborhood system and $L$-fuzzy uniformity.
\end{abstract}

AMS Subject Classification: 03E72, 06A15, 06F07, 54F05

Key Words: complete residuated lattice, $L$-neighborhood space, $L$-fuzzy uniform space, $L$-fuzzy topologies

\section{Introduction}

Lowen [9] introduced the notion of fuzzy uniformities as a viewpoint of the enourage approach. Many researchers $[5-8,12]$ studied the different approach as powerset $[6,12]$ or the uniform covering [5]. Kim [8] introduced the notion of fuzzy uniformities as an extension of Lowen in a stsc-quantale lattice $L$.

Received: September 4, 2014

(c) 2015 Academic Publications, Ltd. url: www.acadpubl.eu

${ }^{\S}$ Correspondence author 
On the other hand, Hájek [4] introduced a complete residuated lattice which is an algebraic structure for many valued logic. Bělohlávek [2] investigated information systems and decision rules in complete residuated lattices.

In this paper, we define $L$-neighborhood spaces and investigated the topological properties of $L$-fuzzy uniformity in complete residuated lattices. We obtain $L$-fuzzy topology and $L$-neighborhood spaces induced by $L$-fuzzy uniformity. Moreover, we study the relations among $L$-fuzzy topology, $L$-neighborhood system and $L$-fuzzy uniformity.

\section{Preliminaries}

Definition 2.1. (see [2], [4]) An algebra $(L, \wedge, \vee, \odot, \rightarrow, \perp, \top)$ is called a complete residuated lattice if it satisfies the following conditions:

(C1) $L=(L, \leq, \vee, \wedge, \perp, \top)$ is a complete lattice with the greatest element $\top$ and the least element $\perp$;

(C2) $(L, \odot, \top)$ is a commutative monoid;

(C3) $x \odot y \leq z$ iff $x \leq y \rightarrow z$ for $x, y, z \in L$.

For $\alpha \in L, \lambda \in L^{X}$, we denote $(\alpha \rightarrow \lambda),(\alpha \odot \lambda), \alpha_{X} \in L^{X}$ as $(\alpha \rightarrow \lambda)(x)=$ $\alpha \rightarrow \lambda(x),(\alpha \odot \lambda)(x)=\alpha \odot \lambda(x), \alpha_{X}(x)=\alpha$.

In this paper, we assume that $(L, \vee, \wedge, \odot, \rightarrow, \perp, \top)$ is a complete residuated lattice.

Lemma 2.2. (see [2], [4]) For each $x, y, z, w, x_{i}, y_{i} \in L$, the following properties hold:

(1) If $y \leq z$, then $x \odot y \leq x \odot z$.

(2) If $y \leq z$, then $x \rightarrow y \leq x \rightarrow z$ and $z \rightarrow x \leq y \rightarrow x$.

(3) $x \rightarrow y=\top$ iff $x \leq y$.

(4) $x \rightarrow \top=\top$ and $\top \rightarrow x=x$.

(5) $x \odot y \leq x \wedge y$.

(6) $x \odot\left(\bigvee_{i \in \Gamma} y_{i}\right)=\bigvee_{i \in \Gamma}\left(x \odot y_{i}\right)$ and $\left(\bigvee_{i \in \Gamma} x_{i}\right) \odot y=\bigvee_{i \in \Gamma}\left(x_{i} \odot y\right)$.

(7) $x \rightarrow\left(\bigwedge_{i \in \Gamma} y_{i}\right)=\bigwedge_{i \in \Gamma}\left(x \rightarrow y_{i}\right)$ and $\left(\bigvee_{i \in \Gamma} x_{i}\right) \rightarrow y=\bigwedge_{i \in \Gamma}\left(x_{i} \rightarrow y\right)$. $\left.y_{i}\right)$.

(8) $\bigvee_{i \in \Gamma} x_{i} \rightarrow \bigvee_{i \in \Gamma} y_{i} \geq \bigwedge_{i \in \Gamma}\left(x_{i} \rightarrow y_{i}\right)$ and $\bigwedge_{i \in \Gamma} x_{i} \rightarrow \bigwedge_{i \in \Gamma} y_{i} \geq \bigwedge_{i \in \Gamma}\left(x_{i} \rightarrow\right.$ 
(9) $(x \rightarrow y) \odot x \leq y$ and $(x \rightarrow y) \odot(y \rightarrow z) \leq(x \rightarrow z)$.

(10) $x \rightarrow y \leq(y \rightarrow z) \rightarrow(x \rightarrow z)$ and $x \rightarrow y \leq(z \rightarrow x) \rightarrow(z \rightarrow y)$.

(11) $(x \odot y) \rightarrow z=x \rightarrow(y \rightarrow z)=y \rightarrow(x \rightarrow z)$.

(12) $y \rightarrow z \leq x \odot y \rightarrow x \odot z$ and $(x \rightarrow z) \odot(y \rightarrow w) \leq x \odot y \rightarrow z \odot w$.

Definition 2.3. (see [3], [10]) Let $X$ be a set. A function $R: X \times X \rightarrow L$ is called an $L$-partial order if it satisfies the following conditions:

(E1) reflexive if $R(x, x)=\top$ for all $x \in X$,

(E2) transitive if $R(x, y) \odot R(y, z) \leq R(x, z)$, for all $x, y, z \in X$,

(E3) antisymmetric if $R(x, y)=R(y, x)=\top$, then $x=y$.

Lemma 2.4. (see [3], [10]) For a given set $X$, define a binary mapping $S: L^{X} \times L^{X} \rightarrow L$ by

$$
S(\lambda, \mu)=\bigwedge_{x \in X}(\lambda(x) \rightarrow \mu(x)) .
$$

Then, for each $\lambda, \mu, \rho, \nu \in L^{X}$, and $\alpha \in L$, the following properties hold:

(1) $S$ is an $L$-partial order on $L^{X}$.

(2) $\lambda \leq \mu$ iff $S(\lambda, \mu) \geq T$,

(3) If $\lambda \leq \mu$, then $S(\rho, \lambda) \leq S(\rho, \mu)$ and $S(\lambda, \rho) \geq S(\mu, \rho)$ for each $\rho \in L^{X}$,

(4) $S(\lambda, \mu) \odot S(\nu, \rho) \leq S(\lambda \odot \nu, \mu \odot \rho)$.

Lemma 2.5. (see [3]) Let $\phi: X \rightarrow Y$ be an ordinary mapping. Define $\phi^{\rightarrow}: L^{X} \rightarrow L^{Y}$ and $\phi^{\leftarrow}: L^{Y} \rightarrow L^{X}$ by

$$
\begin{aligned}
& \phi^{\rightarrow}(\lambda)(y)=\bigvee_{\phi(x)=y} \lambda(x), \quad \forall \lambda \in L^{X}, y \in Y, \\
& \phi^{\leftarrow}(\mu)(x)=\mu(\phi(x))=\mu \circ \phi(x), \quad \forall \mu \in L^{Y} .
\end{aligned}
$$

Then for $\lambda, \mu \in L^{X}$ and $\rho, \nu \in L^{Y}$,

$$
S(\lambda, \mu) \leq S\left(\phi^{\rightarrow}(\lambda), \phi^{\rightarrow}(\mu)\right), \quad S(\rho, \nu) \leq S\left(\phi^{\leftarrow}(\rho), \phi^{\leftarrow}(\nu)\right),
$$

and the equalities hold if $\phi$ is bijective. 
Definition 2.6. (see [5]) A map $\mathcal{T}: L^{X} \rightarrow L$ is called an $L$-fuzzy topology on $X$ if it satisfies the following conditions:

(LO1) $\mathcal{T}\left(\perp_{X}\right)=\mathcal{T}\left(\top_{X}\right)=\top$,

$(\mathrm{LO} 2) \mathcal{T}(\lambda \odot \mu) \geq \mathcal{T}(\lambda) \odot \mathcal{T}(\mu), \quad \forall \quad \lambda, \mu \in L^{X},(\mathrm{LO} 3) \quad \mathcal{T}\left(\bigvee_{i} \lambda_{i}\right) \geq$ $\bigwedge_{i} \mathcal{T}\left(\lambda_{i}\right), \quad \forall\left\{\lambda_{i}\right\}_{i \in \Gamma} \subseteq L^{X}$.

An $L$-fuzzy topology is called enriched if

(R) $\mathcal{T}(\alpha \odot \lambda) \geq \mathcal{T}(\lambda)$ for all $\lambda \in L^{X}$ and $\alpha \in L$.

The pair $(X, \mathcal{T})$ is called an $L$-fuzzy topological space.

Let $\left(X, \mathcal{T}_{1}\right)$ and $\left(Y, \mathcal{T}_{2}\right)$ be two $L$-fuzzy topological spaces. A mapping $\phi$ : $X \rightarrow Y$ is said to be $L$-continuous iff for each $\lambda \in L^{Y}$,

$$
\mathcal{T}_{2}(\lambda) \leq \mathcal{T}_{1}\left(\phi^{\leftarrow}(\lambda)\right)
$$

Definition 2.7. (see [8]) A map $\mathcal{U}: L^{X \times X} \rightarrow L$ is called an $L$-fuzzy uniformity on $X$ iff the following conditions hold:

(LU1) There exists $u \in L^{X \times X}$ such that $\mathcal{U}(u)=\top$.

(LU2) If $v \leq u$, then $\mathcal{U}(v) \leq \mathcal{U}(u)$.

(LU3) For every $u, v \in L^{X \times X}, \mathcal{U}(u \odot v) \geq \mathcal{U}(u) \odot \mathcal{U}(v)$.

(LU4) If $\mathcal{U}(u) \neq \perp$ then $\top_{\triangle} \leq u$ where

$$
\top_{\triangle}(x, y)= \begin{cases}\top, & \text { if } \mathrm{x}=\mathrm{y} \\ \perp, & \text { if } x \neq y\end{cases}
$$

(LU5) $\mathcal{U}(u) \leq \mathcal{U}\left(u^{-1}\right)$, where $u^{-1}(x, y)=u(y, x)$.

(LU6) $\mathcal{U}(u) \leq \bigvee\{\mathcal{U}(v) \mid v \circ v \leq u\}, \quad \forall u \in L^{X \times X}$, where

$$
v \circ v(x, y)=\bigvee_{z \in X} v(x, z) \odot v(z, y), \quad \forall x, y \in X
$$

An $L$-fuzzy uniformity $\mathcal{U}$ on $X$ is said to be stratified if:

(R) $\mathcal{U}(\alpha \odot u) \geq \alpha \odot \mathcal{U}(u), \quad \forall u \in L^{X \times X}$.

The pair $(X, \mathcal{U})$ is called an $L$-fuzzy uniform space. 
Let $(X, \mathcal{U})$ and $(Y, \mathcal{V})$ be $L$-fuzzy uniform spaces, and $\phi: X \rightarrow Y$ be a mapping. Then $\phi$ is said to be $L$-fuzzy uniformly continuous if $\mathcal{V}(v) \leq$ $\mathcal{U}\left((\phi \times \phi)^{\leftarrow}(v)\right)$, for every $v \in L^{Y \times Y}$.

Remark 2.8. Let $(X, \mathcal{U})$ be an $L$-fuzzy uniform space.

(1) By (LU1) and (LU2), we have $\mathcal{U}\left(\top_{X \times X}\right)=\top$ because $u \leq \top_{X \times X}$ for all $u \in L^{X \times X}$.

(2) Since $\mathcal{U}(u) \leq \mathcal{U}\left(u^{-1}\right) \leq \mathcal{U}\left(\left(u^{-1}\right)^{-1}\right)=\mathcal{U}(u)$, then $\mathcal{U}(u)=\mathcal{U}\left(u^{-1}\right)$.

\section{Topological Properties of $L$-Fuzzy Uniform Spaces}

Definition 3.1. A map $N: X \rightarrow L^{L^{X}}$ is called an $L$-neighborhood system on $X$ if $N=\left\{N_{x} \mid x \in X\right\}$ satisfies the following conditions

(LN1) $N_{x}\left(\top_{X}\right)=\top$ and $N_{x}\left(\perp_{X}\right)=\perp$,

(LN2) $N_{x}(\lambda \odot \mu) \geq N_{x}(\lambda) \odot N_{x}(\mu)$ for each $\lambda, \mu \in L^{X}$,

(LN3) If $\lambda \leq \mu$, then $N_{x}(\lambda) \leq N_{x}(\mu)$,

(LN4) $N_{x}(\lambda) \leq \lambda(x)$ for all $\lambda \in L^{X}$.

An $L$-neighborhood system is called stratified if

(R) $N_{x}(\alpha \odot \lambda) \geq \alpha \odot N_{x}(\lambda)$ for all $\lambda \in L^{X}$ and $\alpha \in L$.

The pair $(X, N)$ is called an $L$-neighborhood space.

Let $(X, N)$ and $(Y, M)$ be two $L$-neighborhood spaces. A mapping $\phi: X \rightarrow$ $Y$ is said to be $L$-continuous at $x \in X$ iff $M_{\phi(x)}(\lambda) \leq N_{x}\left(\phi^{\leftarrow}(\lambda)\right)$ for each $\lambda \in L^{Y}, \phi$ is $L$-continuous if it is $L$-continuous at every $x \in X$.

Theorem 3.2. Let $(X, \mathcal{U})$ be an $L$-fuzzy uniform space. Define a map $N^{\mathcal{U}}: X \rightarrow L^{L^{X}}$ by

$$
N_{x}^{\mathcal{U}}(\lambda)=\bigvee_{u} \mathcal{U}(u) \odot S(u[x], \lambda), \quad \forall \lambda \in L^{X}, x \in X,
$$

where $u[x](y)=u(y, x)$. Then the following properties hold:

(1) $\left(X, N^{\mathcal{U}}\right)$ is an $L$-neighborhood space.

(2) If $\mathcal{U}$ is stratified, then $N$ is also stratified. 
Proof. (1) (LN1) For $\mathcal{U}(u) \neq \perp, \top_{\triangle} \leq u$. Then

$$
\begin{aligned}
N_{x}^{\mathcal{U}}\left(\perp_{X}\right) & =\bigvee_{u} \mathcal{U}(u) \odot S\left(u[x], \perp_{X}\right) \\
& \leq \bigvee_{u}(\mathcal{U}(u) \odot(u(x, x) \rightarrow \perp)) \\
& =\bigvee_{u}\left(\mathcal{U}(u) \odot\left(\top_{\triangle}(x, x) \rightarrow \perp\right)\right)=\perp .
\end{aligned}
$$

Hence $N_{x}^{\mathcal{U}}\left(\perp_{X}\right)=\perp$. Also, $N_{x}^{\mathcal{U}}\left(\top_{X}\right)=\top$, because

$$
N_{x}^{\mathcal{U}}\left(\top_{X}\right) \geq \mathcal{U}\left(\top_{X \times X}\right) \odot \bigwedge_{y \in X}\left(\top_{X \times X}(x, y) \rightarrow \top_{X}(y)\right)=\top .
$$

(LN2) By Lemma 2.4 (4), we have

$$
\begin{aligned}
& N_{x}^{\mathcal{U}}(\lambda) \odot N_{x}^{\mathcal{U}}(\mu) \\
& =\left(\bigvee_{u} \mathcal{U}(u) \odot S(u[x], \lambda)\right) \odot\left(\bigvee_{u} \mathcal{U}(v) \odot S(v[x], \mu)\right) \\
& =\bigvee_{u, v} \mathcal{U}(u) \odot \mathcal{U}(v) \odot S(u[x], \lambda) \odot S(v[x], \mu) \\
& \leq \bigvee_{u, v} \mathcal{U}(u \odot v) \odot S((u \odot v)[x], \lambda \odot \mu) \\
& \leq \bigvee_{w} \mathcal{U}(w) \odot S(w[x], \lambda \odot \mu)=N_{x}^{\mathcal{U}}(\lambda \odot \mu)
\end{aligned}
$$

(LN3) By Lemma 2.4 (3), we have

$$
\begin{aligned}
N_{x}^{\mathcal{U}}(\lambda) & =\bigvee_{u} \mathcal{U}(u) \odot S(u[x], \lambda) \\
& \leq \bigvee_{u} \mathcal{U}(u) \odot S(u[x], \mu)=N_{x}^{\mathcal{U}}(\mu)
\end{aligned}
$$

(LN4) For $\mathcal{U}(u) \neq \perp, \top_{\triangle} \leq u$.

$$
N_{x}^{\mathcal{U}}(\lambda)=\bigvee_{u} \mathcal{U}(u) \odot \bigwedge_{y \in X}(u(y, x) \rightarrow \lambda(y))
$$




$$
\leq \bigvee_{u}\{\mathcal{U}(u) \odot(u(x, x) \rightarrow \lambda(x))\} \leq \lambda(x)
$$

This implies that $\left(X, N_{x}^{\mathcal{U}}\right)$ is an $L$-neighborhood space.

$(2)$

$$
\begin{aligned}
& \alpha \odot N_{x}^{\mathcal{U}}(\lambda)=\alpha \odot \bigvee_{u} \mathcal{U}(u) \odot S(u[x], \lambda) \\
& =\bigvee_{u} \alpha \odot \mathcal{U}(u) \odot S(\alpha, \alpha) \odot S(u[x], \lambda) \\
& \leq \bigvee_{u} \mathcal{U}(\alpha \odot u) \odot S(\alpha \odot u[x], \alpha \odot \lambda) \\
& \leq N_{x}^{\mathcal{U}}(\alpha \odot \lambda) .
\end{aligned}
$$

Theorem 3.3. If $\phi:(X, \mathcal{U}) \rightarrow(Y, \mathcal{V})$ is $L$-fuzzy uniformly continuous, then $\phi:\left(X, N^{\mathcal{U}}\right) \rightarrow\left(Y, N^{\mathcal{V}}\right)$ is L-continuous.

Proof. First we show that $\phi^{\leftarrow}(v[\phi(x)])=(\phi \times \phi)^{\leftarrow}(v)[x]$ from

$$
\begin{aligned}
& \phi^{\leftarrow}(v[\phi(x)])(z)=v[\phi(x)](\phi(z))=v(\phi(z), \phi(x)) \\
& =(\phi \times \phi)^{\leftarrow}(v)(z, x)=(\phi \times \phi)^{\leftarrow}(v)[x](z) .
\end{aligned}
$$

Thus, by Lemma 2.5, we have

$$
\begin{aligned}
& S(v[\phi(x)], \lambda) \leq S\left(\phi^{\leftarrow}(v[\phi(x)]), \phi^{\leftarrow}(\lambda)\right) \\
& =S\left((\phi \times \phi)^{\leftarrow}(v)[x], \phi^{\leftarrow}(\lambda)\right) . \\
& N_{\phi(x)}^{\mathcal{V}}(\lambda)=\bigvee_{v} \mathcal{V}(v) \odot S(v[\phi(x)], \lambda) \\
& \leq \bigvee_{v} \mathcal{V}(v) \odot S\left((\phi \times \phi)^{\leftarrow}(v)[x], \phi^{\leftarrow}(\lambda)\right) \\
& \leq \bigvee_{u} \mathcal{U}\left((\phi \times \phi)^{\leftarrow}(v)\right) \odot S\left((\phi \times \phi)^{\leftarrow}(v)[x], \phi^{\leftarrow}(\lambda)\right) \\
& \leq N_{x}^{\mathcal{U}}\left(\phi^{\leftarrow}(\lambda)\right) .
\end{aligned}
$$


Theorem 3.4. (1) The L-neighborhood system $N^{\mathcal{U}}=\left\{N_{x}^{\mathcal{U}} \mid x \in X\right\}$ can be constructed from the cuts $\left\{u \in L^{X \times X} \mid \mathcal{U} \geq \alpha\right\}$ as follows:

$$
N_{x}^{\mathcal{U}}(\lambda)=\bigvee_{\alpha \in L} \alpha \odot N_{x}^{\mathcal{U}}(\lambda, \alpha)
$$

where $N_{x}^{\mathcal{U}}(\lambda, \alpha)=\bigvee_{v}\{S(u[x], \lambda) \mid \mathcal{U}(u) \geq \alpha\}$.

$$
N_{x}^{\mathcal{U}}(\lambda) \leq \bigvee_{v}\left\{N_{x}^{\mathcal{U}}(\rho) \mid \rho(z) \leq N_{z}^{\mathcal{U}}(\lambda, \mathcal{U}(v))\right\}
$$

Proof. (1) If for some $y \in X$ we have $A(y) \geq \alpha$, then we can write $A(y) \odot B(y) \geq \alpha \odot B(y)$ and

$$
\bigvee\{A(x) \odot B(x) \mid A(x) \geq \alpha\} \geq \bigvee\{\alpha \odot B(y) \mid A(x) \geq \alpha\}
$$

Suppose

$$
\bigvee\{A(x) \odot B(x) \mid x \in X\} \not{Z} \bigvee_{\alpha \in L} \bigvee\{\alpha \odot B(x) \mid A(x) \geq \alpha\}
$$

There exists $x_{0} \in X$ such that

$$
A\left(x_{0}\right) \odot B\left(x_{0}\right) \not \leq \bigvee_{\alpha \in L} \bigvee\{\alpha \odot B(x) \mid A(x) \geq \alpha\} .
$$

It is a contradiction. Hence

$$
\bigvee\{A(x) \odot B(x) \mid x \in X\}=\bigvee_{\alpha \in L} \bigvee\{\alpha \odot B(x) \mid A(x) \geq \alpha\}
$$

Applying this equality to the formula giving $N_{x}^{\mathcal{U}}(\lambda)$, we obtain

$$
\begin{aligned}
N_{x}^{\mathcal{U}}(\lambda) & =\bigvee_{\alpha \in L}\{\bigvee \alpha \odot S(u[x], \lambda) \mid \mathcal{U}(u) \geq \alpha\} \\
& =\bigvee_{\alpha \in L}\{\alpha \odot \bigvee\{S(u[x], \lambda) \mid \mathcal{U}(u) \geq \alpha\}\} \\
& =\bigvee_{\alpha \in L}\left\{\alpha \odot N_{x}^{\mathcal{U}}(\lambda, \alpha)\right\} .
\end{aligned}
$$


(2) For $u \in L^{X \times X}$ and $\lambda \in L^{X}$, we have

$$
\begin{aligned}
& N_{x}^{\mathcal{U}}(\lambda)=\bigvee_{u} \mathcal{U}(u) \odot S(u[x], \lambda) \\
& =\bigvee_{u}\left\{\mathcal{U}(u) \odot \bigwedge_{y \in X}(u(y, x) \rightarrow \lambda(y))\right\}(\text { by LU }(6)) \\
& \leq \bigvee_{v}\left\{\mathcal{U}(v) \odot \bigwedge_{y \in X}((v \circ v)(y, x) \rightarrow \lambda(y))\right\} \\
& =\bigvee_{v}\left\{\mathcal{U}(v) \odot \bigwedge_{y \in X}\left(\left(\bigvee_{z \in X} v(z, x) \odot v(y, z)\right) \rightarrow \lambda(y)\right)\right\} \\
& =\bigvee_{v}\left\{\mathcal{U}(v) \odot \bigwedge_{y \in X} \bigwedge_{z \in X}((v(z, x) \odot v(y, z)) \rightarrow \lambda(y))\right\} \\
& =\bigvee_{v}\left\{\mathcal{U}(v) \odot \bigwedge_{y \in X} \bigwedge_{z \in X}(v(z, x) \rightarrow(v(y, z) \rightarrow \lambda(y))\} \quad \text { (by Lemma 2.2 }(12)\right) \\
& =\bigvee_{v}\left\{\mathcal{U}(v) \odot \bigwedge_{z \in X}\left(v(z, x) \rightarrow \bigwedge_{y \in X}(v(y, z) \rightarrow \lambda(y))\right\}\right.
\end{aligned}
$$

Put $\rho(z)=\bigwedge_{y \in X}(v(y, z) \rightarrow \lambda(y))$. Then $\rho(z) \leq N_{z}^{\mathcal{U}}(\lambda, \mathcal{U}(v))$ for all $z \in X$. Thus,

$$
\begin{aligned}
& N_{x}^{\mathcal{U}}(\lambda) \leq \bigvee_{v}\left\{\mathcal{U}(v) \odot \bigwedge_{z \in X}(v(z, x) \rightarrow \rho(z)) \mid \rho(z) \leq N_{z}^{\mathcal{U}}(\lambda, \mathcal{U}(v))\right\} \\
& \leq \bigvee_{v}\left\{N_{x}^{\mathcal{U}}(\rho) \mid \rho(z) \leq N_{z}^{\mathcal{U}}(\lambda, \mathcal{U}(v))\right\} .
\end{aligned}
$$

Theorem 3.5. Let $X$ be any nonempty set and $(Y, M)$ be an $L$ neighborhood space. If $\phi: X \rightarrow Y$ is a mapping, then $(X, N)$ is an $L$-neighborhood space, where

$$
N_{x}(\lambda)=\bigvee\left\{M_{\phi(x)}(\mu) \mid \phi^{\leftarrow}(\mu) \leq \lambda, \mu \in L^{Y}\right\}
$$

Moreover, if $\phi$ is surjective, then $\phi$ is $L$-continuous and if $M$ is stratified, then $N$ is also stratified.

Proof. (LN1) and (LN3) are clearly true.

(LN2) Let $\lambda_{1}, \lambda_{2} \in L^{X}, \mu_{1}, \mu_{2} \in L^{Y}$ and $x \in X$, then we have

$$
N_{x}\left(\lambda_{1}\right) \odot N_{x}\left(\lambda_{2}\right)
$$




$$
\begin{aligned}
& =\bigvee\left\{M_{\phi(x)}\left(\mu_{1}\right) \mid \phi^{\leftarrow}\left(\mu_{1}\right) \leq \lambda_{1}\right\} \odot \bigvee\left\{M_{\phi(x)}\left(\mu_{2}\right) \mid \phi^{\leftarrow}\left(\mu_{2}\right) \leq \lambda_{2}\right\} \\
& =\bigvee\left\{M_{\phi(x)}\left(\mu_{1}\right) \odot M_{\phi(x)}\left(\mu_{2}\right) \mid \phi^{\leftarrow}\left(\mu_{1}\right) \leq \lambda_{1}, \phi^{\leftarrow}\left(\mu_{2}\right) \leq \lambda_{2}\right\} \\
& \leq \bigvee\left\{M_{\phi(x)}\left(\mu_{1} \odot \mu_{2}\right) \mid \phi^{\leftarrow}\left(\mu_{1}\right) \odot \phi^{\leftarrow}\left(\mu_{2}\right) \leq \lambda_{1} \odot \lambda_{2}\right\} \\
& =\bigvee\left\{M_{\phi(x)}(\mu) \mid \phi^{\leftarrow}(\mu) \leq \lambda_{1} \odot \lambda_{2}\right\} \\
& =N_{x}\left(\lambda_{1} \odot \lambda_{2}\right) .
\end{aligned}
$$

(LN4) For any $\lambda \in L^{X}, \mu \in L^{Y}$ and $x \in X$, we have

$$
\begin{aligned}
& N_{x}(\lambda)=\bigvee\left\{M_{\phi(x)}(\mu) \mid \phi^{\leftarrow}(\mu) \leq \lambda\right\} \leq \bigvee\left\{\mu\left(\phi^{\rightarrow}(x)\right) \mid \phi^{\leftarrow}(\mu) \leq \lambda\right\} \\
& =\bigvee\left\{\phi^{\leftarrow}(\mu)(x) \mid \phi^{\leftarrow}(\mu) \leq \lambda\right\} \leq \lambda(x) .
\end{aligned}
$$

Now, since the mapping $\phi:(X, N) \rightarrow(Y, M)$ is $L$-continuous and if $x \in$ $X, \mu \in L^{Y}$, it follows that

$$
\begin{aligned}
& N_{x}\left(\phi^{\leftarrow}(\mu)\right)=\bigvee\left\{M_{\phi(x)}(\lambda) \mid \phi^{\leftarrow}(\lambda) \leq \phi^{\leftarrow}(\mu)\right\} \\
& \geq \bigvee\left\{M_{\phi(x)}(\lambda) \mid \lambda \leq \mu\right\} \geq M_{\phi(x)}(\mu) .
\end{aligned}
$$

Finally, if $M$ is stratified, then $N$ is also stratified. In fact, for any $\alpha \in L$ and $\lambda \in L^{X}$, we have

$$
\begin{aligned}
& \alpha \odot N_{x}(\lambda)=\alpha \odot \bigvee\left\{M_{\phi(x)}(\mu) \mid \phi^{\leftarrow}(\mu) \leq \lambda\right\} \\
& \leq \bigvee\left\{\alpha \odot M_{\phi(x)}(\mu) \mid \alpha \odot \phi^{\leftarrow}(\mu) \leq \alpha \odot \lambda\right\} \\
& \leq \bigvee\left\{M_{\phi(x)}(\alpha \odot \mu) \mid \phi^{\leftarrow}(\alpha \odot \mu) \leq \alpha \odot \lambda\right\} \\
& =N_{x}(\alpha \odot \lambda) .
\end{aligned}
$$

Theorem 3.6. Let $(X, N)$ be a L-neighborhood space. Define a map $\mathcal{T}_{N}: L^{X} \rightarrow L$ by

$$
\mathcal{T}_{N}(\lambda)=\bigwedge_{x \in X}\left(\lambda(x) \rightarrow N_{x}(\lambda)\right)
$$


Then (1) $\mathcal{T}_{N}$ is an L-fuzzy topology on $X$,

(2) If $N_{x}$ is stratified, then $\mathcal{T}_{N}$ is an enriched $L$-fuzzy topology.

Proof. (1) (LO1)

$$
\begin{aligned}
& \mathcal{T}_{N}\left(\top_{X}\right)=\bigwedge_{x \in X}\left(\top_{X}(x) \rightarrow N_{x}\left(\top_{X}\right)\right)=\top \rightarrow \top=\top, \\
& \mathcal{T}_{N}\left(\perp_{X}\right)=\bigwedge_{x \in X}\left(\perp_{X}(x) \rightarrow N_{x}\left(\perp_{X}\right)\right)=\perp \rightarrow \perp=\perp .
\end{aligned}
$$

(LO2)

$$
\begin{aligned}
& \mathcal{T}_{N}(\lambda \odot \mu)=\bigwedge_{x \in X}\left((\lambda \odot \mu)(x) \rightarrow N_{x}(\lambda \odot \mu)\right) \\
& \geq \bigwedge_{x \in X}\left((\lambda \odot \mu)(x) \rightarrow\left(N_{x}(\lambda) \odot N_{x}(\mu)\right)\right)
\end{aligned}
$$

(by Lemma $2.2(12)$ )

$$
\begin{aligned}
& \geq \bigwedge_{x \in X}\left(\lambda(x) \rightarrow N_{x}(\lambda)\right) \odot \bigwedge_{x \in X}\left(\mu(x) \rightarrow N_{x}(\mu)\right) \\
& =\mathcal{T}_{N}(\lambda) \odot \mathcal{T}_{N}(\mu) .
\end{aligned}
$$

(LO3)

$$
\begin{aligned}
\mathcal{T}_{N}\left(\bigvee_{i} \lambda_{i}\right)= & \bigwedge_{x \in X}\left(\left(\bigvee_{i} \lambda_{i}\right)(x) \rightarrow N_{x}\left(\bigvee_{i} \lambda_{i}\right)\right) \\
\geq & \bigwedge_{x \in X}\left(\left(\bigvee_{i} \lambda_{i}\right)(x) \rightarrow \bigvee_{i} N_{x}\left(\lambda_{i}\right)\right) \\
& (\text { by Lemma } 2.2(8)) \\
\geq & \bigwedge_{i} \bigwedge_{x \in X}\left(\lambda_{i}(x) \rightarrow N_{x}\left(\lambda_{i}\right)\right)=\bigwedge_{i} \mathcal{T}_{N}\left(\lambda_{i}\right) .
\end{aligned}
$$

(2) By Lemma 2.2 (12), we have

$$
\begin{aligned}
& \mathcal{T}_{N}(\alpha \odot \lambda)=\bigwedge_{x \in X}\left((\alpha \odot \lambda)(x) \rightarrow N_{x}(\alpha \odot \lambda)\right) \\
& \geq \bigwedge_{x \in X}\left((\alpha \odot \lambda)(x) \rightarrow\left(\alpha \odot N_{x}(\lambda)\right)\right)
\end{aligned}
$$




$$
\geq \bigwedge_{x \in X}\left(\lambda(x) \rightarrow N_{x}(\lambda)\right)=\mathcal{T}_{N}(\lambda)
$$

Corollary 3.7. Let Let $(X, \mathcal{U})$ be an L-fuzzy uniform space and $\left\{N_{x}^{\mathcal{U}} \mid x \in\right.$ $X\}$ be an $L$-neighorhood system on $X$. Define a map $\mathcal{T}_{\mathcal{U}}: L^{X} \rightarrow L$ by

$$
\mathcal{T}_{\mathcal{U}}(\lambda)=\bigwedge_{x \in X}\left(\lambda(x) \rightarrow N_{x}^{\mathcal{U}}(\lambda)\right)
$$

Then:

(1) $\mathcal{T}_{\mathcal{U}}$ is an L-fuzzy topology on $X$,

(2) If $N_{x}^{\mathcal{U}}$ is stratified, then $\mathcal{T}_{\mathcal{U}}$ is an enriched L-fuzzy topology.

Theorem 3.8. Let $(X, \mathcal{U})$ and $(Y, \mathcal{V})$ be two $L$-fuzzy uniform spaces. If a map $\phi:(X, \mathcal{U}) \rightarrow(Y, \mathcal{V})$ is L-fuzzy uniformly continuous, then a map $\phi:\left(X, \mathcal{T}_{\mathcal{U}}\right) \rightarrow\left(Y, \mathcal{T}_{\mathcal{V}}\right)$ is $L$ - continuous.

Proof.

$$
\begin{aligned}
& \mathcal{T} \mathcal{V}(\lambda) \rightarrow \mathcal{T}_{\mathcal{U}}\left(\phi^{\leftarrow}(\lambda)\right) \\
& =\bigwedge_{y \in Y}\left(\lambda(y) \rightarrow N_{y}^{\mathcal{V}}(\lambda)\right) \rightarrow \bigwedge_{x \in X}\left(\phi^{\leftarrow}(\lambda)(x) \rightarrow N_{x}^{\mathcal{U}}\left(\phi^{\leftarrow}(\lambda)\right)\right) \\
& \geq \bigwedge_{x \in X}\left(\phi^{\leftarrow}(\lambda)(x) \rightarrow N_{\phi^{\leftarrow}(x)}^{\mathcal{V}}(\lambda)\right) \rightarrow \bigwedge_{x \in X}\left(\phi^{\leftarrow}(\lambda)(x) \rightarrow N_{x}^{\mathcal{U}}\left(\phi^{\leftarrow}(\lambda)\right)\right) \\
& \geq \bigwedge_{x \in X}\left(\left(\phi^{\leftarrow}(\lambda) \rightarrow N_{\phi^{\leftarrow}(x)}^{\mathcal{V}}(\lambda)\right) \rightarrow\left(\phi^{\leftarrow}(\lambda) \rightarrow N_{x}^{\mathcal{U}}\left(\phi^{\leftarrow}(\lambda)\right)\right)\right)
\end{aligned}
$$

(by Lemma $2.2(8)$ )

$$
\left.\geq \bigwedge_{x \in X}\left(N_{\phi^{\leftarrow}(x)}^{\mathcal{V}}(\lambda) \rightarrow N_{x}^{\mathcal{U}}\left(\phi^{\leftarrow}(\lambda)\right)\right) \text { (by Lemma } 2.2(10)\right) \text {. }
$$

Thus, if $N_{\phi^{\leftarrow}(x)}^{\mathcal{V}}(\lambda) \leq N_{x}^{\mathcal{U}}\left(\phi^{\leftarrow}(\lambda)\right)$, then $\mathcal{T}_{\mathcal{V}}(\lambda) \leq \mathcal{T}_{\mathcal{U}}\left(\phi^{\leftarrow}(\lambda)\right)$. So, $\phi$ is $L$-continuous.

Example 3.9. Let $(L=[0,1], \odot, \rightarrow)$ be a complete residuated lattice defined by

$$
x \odot y=(x+y-1) \vee 0, x \rightarrow y=(1-x+y) \wedge 1 .
$$


Let $X=\{x, y, z\}$ be a set and $w, w \odot w \in L^{X \times X}$ such that

$$
w=\left(\begin{array}{ccc}
1 & 0.7 & 0.6 \\
0.7 & 1 & 0.3 \\
0.6 & 0.3 & 1
\end{array}\right), w \odot w=\left(\begin{array}{ccc}
1 & 0.4 & 0.2 \\
0.4 & 1 & 0 \\
0.2 & 0 & 1
\end{array}\right)
$$

Define $\mathcal{U}: L^{X} \rightarrow L$ as follows

$$
\mathcal{U}(u)= \begin{cases}1, & \text { if } u=1_{X \times X}, \\ 0.6, & \text { if } w \leq u \neq 1_{X \times X}, \\ 0.3, & \text { if } w \odot w \leq u \geq w, \\ 0, & \text { otherwise. }\end{cases}
$$

Since $0.3=\mathcal{U}(w \odot w) \geq \mathcal{U}(w) \odot \mathcal{U}(w)=0.2$ and $w \circ w=w,(w \odot w) \circ(w \odot w)=$ $(w \odot w), w=w^{-1}, \mathcal{U}$ is an $L$-fuzzy uniformity on $X$.

$$
\begin{gathered}
N_{x}^{\mathcal{U}}(\lambda)=\bigvee_{u} \mathcal{U}(u) \odot S(u[x], \lambda), \quad \forall \lambda \in L^{X}, x \in X \\
N_{x}^{\mathcal{U}}(\lambda)=\bigvee_{u} \mathcal{U}(u) \odot S(u[x], \lambda) \\
=(\lambda(x) \wedge \lambda(y) \wedge \lambda(z)) \vee(0.6 \odot(\lambda(x) \wedge(0.3+\lambda(y)) \wedge(0.4+\lambda(z))) \\
\vee(0.3 \odot(\lambda(x) \wedge(0.6+\lambda(y)) \wedge(0.8+\lambda(z))) \\
N_{y}^{\mathcal{U}}(\lambda)=(\lambda(x) \wedge \lambda(y) \wedge \lambda(z)) \vee(0.6 \odot((0.3+\lambda(x)) \wedge \lambda(y) \wedge(0.7+\lambda(z))) \\
\vee(0.3 \odot((0.6+\lambda(x)) \wedge \lambda(y))) \\
N_{z}^{\mathcal{U}}(\lambda)=(\lambda(x) \wedge \lambda(y) \wedge \lambda(z)) \vee(0.6 \odot((0.4+\lambda(x)) \wedge(0.7+\lambda(y)) \wedge \lambda(z))) \\
\vee(0.3 \odot((0.8+\lambda(x)) \wedge \lambda(z)))
\end{gathered}
$$

For $\lambda=(0.4,0.6,0.8)$,

$$
\begin{gathered}
N_{x}^{\mathcal{U}}(\lambda)=0.4, N_{y}^{\mathcal{U}}(\lambda)=0.4, N_{z}^{\mathcal{U}}(\lambda)=0.4 \\
\mathcal{T}_{N}(\lambda)=\bigwedge_{x \in X}\left(\lambda(x) \rightarrow N_{x}(\lambda)\right)=0.7 .
\end{gathered}
$$

For $\rho=(0.8,0.2,0.7)$,

$$
\begin{gathered}
N_{x}^{\mathcal{U}}(\lambda)=0.2, N_{y}^{\mathcal{U}}(\lambda)=0.2, N_{z}^{\mathcal{U}}(\lambda)=0.3 \\
\mathcal{T}_{N}(\lambda)=\bigwedge_{x \in X}\left(\lambda(x) \rightarrow N_{x}(\lambda)\right)=0.4 .
\end{gathered}
$$




\section{References}

[1] R. Badard, A.A. Ramadan, A.S. Mashhour, Smooth preuniform and proximity spaces, Fuzzy Sets and Systems, 59 (1993), 95-107.

[2] R. Bělohlávek,Fuzzy Relational Systems, Kluwer Academic Publishers, New York , (2002), doi: 10.1007/978-1-4615-0633-1.

[3] Fang Jinming, I-fuzzy Alexandrov topologies and specialization orders, Fuzzy Sets and Systems, 158(2007), 2359-2374, doi: 10.1016/j.fss.2007.05.001.

[4] P. Hájek, Metamathematices of Fuzzy Logic, Kluwer Academic Publishers, Dordrecht (1998), doi: 10.1007/978-94-011-5300-3.

[5] U. Höhle, S.E. Rodabaugh, Mathematics of Fuzzy Sets: Logic, Topology, and Measure Theory, The Handbooks of Fuzzy Sets Series 3, Kluwer Academic Publishers, Boston, 1999, doi: 10.1007/978-1-4615-5079-2.

[6] B. Hutton, Uniformities in fuzzy topological spaces, J. Math. Anal. Appl., 58 (1977), 74-79. doi: 10.1016/0022-247x(77)90192-5.

[7] A.K. Katsaras, On fuzzy uniform spaces, J. Math. Anal. Appl., 101, 1984, 97-113. doi: 10.1016/0022-247x(84)90060-x.

[8] Y.K. Kim, A.A. Ramadan, M. A. Usama, L-fuzzy Uniform Spaces, The Journal of Fuzzy Mathematics, 14 (2006), 821-850.

[9] R. Lowen, Fuzzy uniform spaces, J. Math. Anal. Appl., 82 (1981), 370-385, doi: $10.1016 / 0022-247 x(81) 90202-x$.

[10] H. Lai, D. Zhang, Fuzzy preorder and fuzzy topology, Fuzzy Sets and Systems, 157 (2006), 1865-1885, doi: 10.1016/j.fss.2006.02.013.

[11] A.A. Ramadan,Y.C. Kim, M.K. El-Gayyar, On fuzzy uniform spaces, The Journal of Fuzzy Mathematics, 11 (2003), 279-299.

[12] S.E. Rodabaugh, E.P. Klement, Topological and Algebraic Structures In Fuzzy Sets, The Handbook of Recent Developments in the Mathematics of Fuzzy Sets, Kluwer Academic Publishers, Boston, Dordrecht, London, 2003, doi: 10.1007/978-94-017-0231-7. 